\title{
ENERGÍAS RENOVABLES Y CAMBIO CLIMÁTICO
}

Pedro Gamio Aita

\section{Resumen}

El presente trabajo evalúa las características de una estrategia nacional que enfrente al cambio climático en el Perú, abordando aspectos económicos, sociales y ambientales, incidiendo en el uso de las energías renovables y señalando las limitaciones que recortan las posibilidades de respuesta y gestión eficiente frente los problemas de degradación ambiental: contaminación del agua y del aire, desastres naturales, degradación de suelos, deforestación, acumulación de residuos sólidos, entre otros. Enfatiza el uso y potencialidad de las energías renovables, principalmente la hidroenergía, la eólica, el gas natural, la geotermia, la energía solar.

Palabras clave: energias renovables, planificación estratégica ambiental, degradación ambiental, cambio climático, estrategia de mitigación.

\section{Abstract}

This paper evaluates the characteristics of a national strategy confront to climate change in Peru, taking into account the economic, social and environmental aspects, with particular attention in the use of renewable energy sources and pointing constraints that reduce the chances of response and efficient management to environmental degradation problems: water and air pollutions, natural disasters, soil degradation, deforestation, accumulation of waste, among others. Emphasizes the use and potential of renewable, mostly hydroenergy, wind, natural gas, geothermal energy, solar energy.

Key words: renewable energy, environmental strategic planning, environmental degradation, climate change, mitigation strategy. 


\section{Energias renovables y estrategia nacional frente al cambio climático en el Perú}

En la vida diaria se encuentran evidencias a lo largo y ancho del Perú de una controvertida situación ambiental, que agudiza el cuadro de los 8'300,000 peruanos en condición de pobreza, contaminación creada por la actividad humana, a lo que se suman los efectos del calentamiento global. El Perú enfrenta un gran desafio. Una sociedad que busca su desarrollo debe manejar mejor y conocer los recursos fisicos, naturales, culturales y sociales que lo componen. Es un imperativo el desarrollo de la planificación estratégica socio ambiental en las actividades económicas. En el Perú falta pensar en el mediano y largo plazo, falta articular políticas públicas a favor de un ambiente sano $y$ desarrollo sustentable.

En sintesis, el país enfrenta problemas de falta de institucionalidad que limitan la posibilidad de respuesta y gestión eficiente frente a la contaminación y deterioro creciente de sus ecosistemas. Este deterioro ambiental compromete a la fecha el 4\% del PBI (Banco Mundial), afecta a los espacios rurales $\mathrm{y}$ entornos urbanos, que sufren la degradación ambiental vinculada principalmente a la contaminación del agua, la contaminación del aire en exteriores e interiores, los desastres naturales, la degradación de suelos, la deforestación, la acumulación de residuos sólidos, entre otros.
A estos aspectos ambientales netamente locales, se debe sumar los efectos del cambio climático, los cuales se proyectan en $4.5 \%$ de pérdida del PBI al 2025 (Andina, 2008). Por ello, un aspecto relevante es la vulnerabilidad de nuestro país frente a los impactos del cambio climático, que lo coloca entre los países más vulnerables en el mundo. Actualmente su impacto ya se siente. Los glaciares han retrocedido un $22 \%$ en los últimos 30 años, afectando nuestra disponibilidad de agua a futuro. Los modelos de escenarios climáticos indican que el Fenómeno del Niño podrá ser más intenso y más frecuente. Se ha percibido un aumento en la recurrencia de sequías y heladas en cuencas de gran importancia por ser proveedoras de alimentos del país.

Una estrategia de mitigación debe apoyarse en co-beneficios locales y sinergias con el crecimiento económico, la gestión de los recursos renovables y no renovables, sus impactos sobre la calidad ambiental local, y sobre la política de adaptación; lado a lado con una política proactiva de mitigación global que use la propuesta peruana de mitigación -que el Perú debe ejecutar- para reducir el impacto que el Perú sufre al cambiar su régimen climático. En este sentido, un esfuerzo de mitigación local se apoyaría en una política exterior de promoción de la mitigación global.

\section{Estrategia Nacional de Mitigación de Emisiones de GEI}

Hay tres líneas de acción a considerar, 
1. La integración de la politica de mitigación dentro de un esfuerzo internacional, con metas ambiciosas de mitigación.

2. Un enfoque en las áreas en las que existen cobeneficios en términos de crecimiento económico, mejoras ambientales locales y de adaptación

3. Un indispensable incremento en la capacidad del Estado y la administración pública de enfrentar el problema y de la sociedad civil peruanade percibirlo y actuar en consecuencia, como aliado estratégico.

La estrategia de mitigación considera una linea base de acción, y un conjunto de politicas y medidas de reducción que se agruparian en programas, o medidas nacionales apropiadas de mitigación (MENAMAs), coordinadas por el Ministerio del Ambiente con participación de los ministerios y organizaciones involucradas, y la sociedad civil. Las MENAMAs agruparian medidas por sectores, contabilizando y registrando en registros ad-hoc todas las emisiones. Utilizarian para su monitoreo regular una versión mejorada del sistema nacional de inventarios, en línea con la propuesta para la creación de dicho sistema.

Los mecanismos de verificación y registro servirian para que terceros puedan verificar dichas reducciones y podrian formar parte de metas voluntarias verificables. En particular, dichas MENAMAs combinarian los proyectos que se pueden ejecutar como MDLs, ya sea como MDLs tradicionales o programáticos; aquellos que se podrían ejecutar con algún apoyo externo adicional, y aquellos que el país realiza por su cuenta, como una contribución a la mitigación global, a cambio de un incremento en la mitigación global. Los MENAMAS combinan medidas e incentivos con proyectos y actividades generadas por ellos. Debido a esta mezcla, deben precisar las acciones que se encuentran dentro del escenario de acción usual $\mathrm{y}$ aquellas otras que representan un esfuerzo adicional. En todos los casos, estos MENAMAs deberían considerar un esquema sólido de coordinación e implementación de políticas, y otro de monitoreo, reporte y verificación. Esto asegura que sus contenidos se ejecutan, y que sus resultados pueden ser seguidos y verificados con el nivel de rigor requerido en cada caso. Al incluir proyectos, permitiría además vender las reducciones generadas en los mercados relevantes de carbono.

Una de las medidas nacionales apropiadas de mitigación, es la de energía, que junto con los sectores forestal y de uso de suelos tienen un lugar central en la estrategia, y debieran ser los primeros en ser desarrollados. Cada MENAMA debe ser desarrollado y encontrarse articulado a la estrategia nacional de mitigación.

\section{Futura matriz energética incorporando fuentes renovables}

La estrategia de mitigación debe posibilitar una más agresiva diversificación de la matriz energética 
con metas precisas en el corto, mediano y largo plazo, donde los instrumentos de gestión ambiental se articulen con la promoción y desarrollo de una economia baja en carbono, fomentando el uso de energías limpias en el desarrollo de cualquier emprendimiento. Para ello, tiene que existir relación y coherencia con la política tributaria, regulatoria y de ordenamiento territorial y zonificación ecológica. Aquí, la tarea corresponde a los tres niveles de gobierno: nacional, regional y local, pero compromete al conjunto de la sociedad civil, que ha mostrado ser más creativa e innovadora que el Estado, cuando se trata de acceder a la energía utilizando energías renovables.

La estrategia se plantea también con el objetivo de poner al Perú en una posición de avanzada en la negociación internacional, incrementando su capacidad de influencia para que otros grandes emisores reduzcan sus emisiones $\mathrm{y}$, en consecuencia los impactos futuros de sus emisiones sobre el clima del país. Además de buscar competitividad y sostenibilidad en el mediano y largo plazo.

La estrategia sugiere privilegiar los esfuerzos de mitigación en aspectos en los cuales el Perú cuenta con activos valiosos en el escenario mundial de mitigación como la amazonia, junto a otros en los cuales se combinan potenciales significativos de reducción con crecimiento económico, cobeneficios y beneficios locales. En esto, la política debe enfocarse en los sectores con mayores oportunidades de reducción a un menor costo, como es el caso específico de la energía.

En paralelo, la estrategia sugiere desarrollar medidas y políticas para mejorar la capacidad de monitoreo, evaluación y verificación de las reducciones, incrementar la percepción de la población sobre el problema y la capacidad del Estado y la administración pública de implementarlas.

La meta propuesta es lograr repartir proporcionalmente para el año 2025 (50\% c/u) la participación de hidrocarburos y las energías renovables. Esto nos asegurara un desarrollo sostenible, mejorando la calidad de vida de la gente $\mathrm{y}$ democratizando gradualmente el uso y acceso a la energía.

1. Uso y potencialidad de las energias renovables

Se denomina energías renovables a la energía que se obtiene de fuentes naturales virtualmente inagotables, unas por la inmensa cantidad de energía que contienen, y otras porque son capaces de regenerarse por medios naturales. Las energías renovables son la mejor opción para dejar de usar petróleo, porque es un recurso contaminante, cada vez más limitado, caro y agotable, porque su empleo en mayor o menor grado origina Gases de Efecto Invernadero que contribuyen a acelerar el Cambio Climático en el Planeta. Solo en Perú, de acuerdo a las cifras del Balance Nacional de Energia, se liberan alrededor de 2550 Ton/hora de $\mathrm{CO} 2$ debido al Consumo de Energía de Combustibles Fósiles. 
La energía renovable más económica en el Perú es la Hidroenergia. Su potencial técnico es alrededor de 8 veces la potencia instalada actual, que al 2008 alcanzaba los $7158 \mathrm{MW}$, siendo sus costos de generación competitivos con la generación térmica. Otra fuente que a futuro se aprecia muy competitiva es la energía eólica, la cual en Perú tiene un potencial aprovechable de cerca de 3 veces la potencia instalada actual. Además, tenemos la Geotermia, Solar fotovoltaica y fototérmica y la Bioenergía.

Las Energías renovables tienen los siguientes efectos positivos:

a) Son intensivas en mano de obra por lo que generan empleo.

b) Sirven para estimular la economía del país a partir del desarrollo de un mercado con alta incidencia en la generación de empleo y en el desarrollo de infraestructura.

b) Contribuyen a mitigar los efectos del cambio climático.

c) Contribuyen a diversificar la matriz energética del país y a mejorar la seguridad energética.

d) Son inagotables, por tanto pueden ser utilizadas permanentemente.

e) Complementan eficazmente el Plan de Electrificación rural, dando energía a muchos pueblos aislados, donde no llega la red convencional.

f) Nos permiten ser un país más competitivo y acceder a mercados internacionales sin restricciones.

En el país, no obstante la importante penetración del gas natural, todavia tenemos una fuerte dependencia al petróleo. El año 2008 importamos 2500 millones de dólares en petróleo y diesel. Esto no guarda coherencia con nuestro potencial de energías renovables y gas natural; resulta una contradicción y una muestra de ineficiencia, no solo desde la perspectiva del cambio climático, la estrategia nacional de mitigación y la sostenibilidad del modelo, sino desde el punto de vista económico, de la competitividad del país.

Según los Mapas Eólicos del Perú, calculados a 50, 80 y 100 mts respectivamente, las zonas con mayor potencial para generación eólica de gran capacidad están en la costa: en las regiones de Piura, Lambayeque e Ica es donde se aprecia mayores velocidades de viento promedio anual. La energía eólica puede complementar a la hidráulica, por cuanto es precisamente el periodo de estiaje el mejor momento de los vientos en la costa peruana, los mismos que tienen una vocación energética por su estabilidad y potencia.

\section{Energía solar}

Según el Atlas de energía solar del Perú (SENAMHI, 2003), la zona de mayor potencial de energía solar del país se encuentra en la costa sur, en las regiones de Arequipa, Moquegua y Tacna (entre los $16^{\circ}$ y $18^{\circ}$ de latitud sur), donde el promedio anual de energía solar incidente diaria estaría en un rango de 6,0 a $6,5 \mathrm{~kW} . \mathrm{h} / \mathrm{m} 2$. Otras zonas con alta disponibilidad de energía solar diaria, entre 5,5 a $6,0 \mathrm{~kW} . \mathrm{h} / \mathrm{m} 2$, se encuentran en la costa norte, en las regiones de Piura y Tumbes (entre los $3^{\circ}$ y $8^{\circ}$ de latitud 
sur), y en gran parte de la sierra, sobre los $2500 \mathrm{msnm}$.

La zona de menores valores de energía solar en el Perú es la selva, en las regiones de Loreto, Ucayali y Madre de Dios, donde se registran valores de 4,5 a $5,0 \mathrm{~kW} . \mathrm{h} / \mathrm{m} 2$, con una zona de minimos valores en el norte de la región Loreto (entre los $0^{\circ}$ y $2^{\circ}$ de latitud sur). No obstante, la alta dispersión de las poblaciones en estas zonas y su particular geografia (escasez de caídas hidráulicas y de recurso eólico) determinan que muchas veces la energía solar fotovoltaica sea la opción más conveniente, a pesar de la menor disponibilidad.

Según el mes del año y la ubicación geográfica, la energía solar diaria acumulada se encuentra en un rango de 2 a $8 \mathrm{~kW} . \mathrm{h} / \mathrm{m} 2$. Sobre esta base, se podría afirmar que, la media nacional rondaría los $5 \mathrm{~kW} \cdot \mathrm{h} / \mathrm{m} 2$. Esto es importante si se considera que, valores iguales o superiores a 4 $\mathrm{kW} . \mathrm{h} / \mathrm{m} 2$ hacen atractivo el uso de tecnologías de conversión fotovoltaica (CER UNI, 2005b). Por otra parte, una característica muy importante de la energía solar en el Perú es su constancia durante el año: los promedios mensuales no varian más del 20\% (Horn, 2007).

Además del Atlas de energía solar, se pueden resaltar otras importantes evaluaciones del potencial de la energía solar en el Perú: Radiación solar en el Perú, de C. Kadono, de la UNI en 1972; Estimación de la energía solar en el Perú, de J. Vásquez, publicado por OLADE en 1987; Tabla de radiación promedio anual, elaborado por OLADE en 1992; y el Atlas de energía solar del Perú, publicado por el SENAMHI y el MEM, en el marco del Proyecto Electrificación rural a base de energía fotovoltaica en el Perú. Cabe mencionar, además, los trabajos de medición de energía solar realizadas a principios de la década del 80 por el propio SENAMHI, el IGP, la UNI, la UNALM, el ITINTEC y la ONERN (CENERGIA, 2004).

\section{Biomasa}

La Biomasa es una fuente de Energía Renovable llamada Bioenergía, que se obtiene a partir de residuos agrícolas, agroindustriales, forestales y urbanos. Su aprovechamiento como energía final se da de tres formas: (1) Como fuente de calor; (2) Como Energía Eléctrica (3) Como Fuerza Motriz para su uso en el transporte. La Bioenergía puede obtenerse de forma Gaseosa, Sólida y Liquida, a través de los llamados Biocombustibles, e involucra una serie de materias primas, tecnologias, productos y equipamiento.

En general, puede decirse que el potencial de biomasa existente en el país para usos energéticos no está debidamente valorizado. Ello debido a que no se han actualizado estudios que evalúen dicho potencial y que incluso los existentes no evalúan la real disponibilidad del recurso bajo criterios de sostenibilidad, es decir, considerando salvaguardar la seguridad alimentaria, evitando la deforestación y el empobrecimiento de la calidad de nutrientes del suelo, entre otros aspectos. Sin embargo, 
según el estudio desarrollado en 1988 por el Programa de las Naciones Unidas para el Desarrollo (PNUD) respecto al Plan de Desarrollo de las Energías Renovables, el potencial teórico anual estimado de los recursos energéticos de la biomasa en el Perú, son los siguientes:

- Forestal:

- Bosques de libre disponibilidad (36,8 millones de $\mathrm{Ha})$ : 767580 GW.h/año.

- Residuos:

- Agrícolas: 8048 GW.h/año.

- Pecuarios: 13235 GW.h/ año. Vacuno: 4800 GW.h/ año. Urbano (4 millones de personas): $2908 \mathrm{GW} . \mathrm{h} /$ año.

- Agroindustriales: Bagazo de caña: 4700 GW.h/año. Cáscara de arroz: 710 GW.h/ año. Residuos de aserraderos: 372 GW.h/año.

La biomasa como fuente de energía tiene posibilidades de utilizarse en el sistema interconectado y en sistemas aislados donde no hay otros recursos renovables. Existen tres grandes regiones donde la biomasa presenta un interesante potencial para ser usada con fines energéticos de mediana $y$ gran potencia (Green Energy, 2005): la costa norte (bagazo de caña, cascarilla de arroz, residuos hidrobiológicos); la selva alta (cascarilla de café, residuos forestales); y la selva baja (residuos forestales). Respecto al consumo de leña, en la costa norte una gran proporción proviene de los bosques secos del norte. En la región de la sierra, considerada por la FAO como región en situación de escasez aguda de biomasa (Horta, 1988), los ecosistemas naturales usualmente utilizados como fuente de energía son los bosques de queñuales y otras formaciones boscosas como los totorales y los yaretales. En esta región las plantaciones de eucalipto han contribuido parcialmente en la solución del problema energético de la región, lo que ha impulsado la realización de programas intensivos de reforestación con fines energéticos. Finalmente, en la selva, la abundancia de biomasa permite afirmar que su consumo no tiene restricción.

\section{Geotermia}

El Perú forma parte del Círculo de Fuego del Pacífico, zona caracterizada por la ocurrencia de movimientos sísmicos, fenómenos tectónicos y elevada concentración de flujo tectónico. Por ello hay en el país numerosas fuentes termales con temperaturas entre $40^{\circ}$ a $90^{\circ}$ C, ubicadas principalmente en la Cordillera Occidental de los Andes y en el Altiplano Sur. Según OLADE, el Perú tendría 156 zonas geotérmicas identificadas; se han reconocido además más de 200 vertientes de agua caliente, así como fumarolas y algunos geysers con temperaturas cercanas a los $100^{\circ} \mathrm{C}$ (Aguinaga, 2006; Coviello, 2006; Battocletti, 1999).

El mayor potencial geotérmico del Perú se encuentra en 6 regiones denominadas geotérmicas (MEM, 2002):

- Región I: Cajamarca (en el departamento del mismo nombre). Región II. Huaraz (en 
Ancash y La Libertad). Región III: Churin (en Lima, Pasco y Huánuco). Región IV: Central (en Huánuco, Huancavelica y Ayacucho). Región V: Cadena de conos volcánicos (en Ayacucho, Apurimac, Arequipa, Moquegua y Tacna). Región VI: Puno y Cusco (en departamentos del mismo nombre).

Las principales áreas de interés serían (Aguinaga, 2006; Coviello, 2006):

- En la región V: Challapalca (en Tacna y Puno); Tutupaca (en Tacna y Moquegua); Calacoa (en Moquegua); Laguna Salinas - Chivay (en Arequipa). (En Challapalca se habría registrado en 1988 un acuífero profundo $\operatorname{con} 270^{\circ} \mathrm{C}$ ).

- En las regiones I y II: Callejón (en Ancash); Otuzco y La Gramma (en La Libertad y Cajamarca); y Cajamarca (en Cajamarca).

Entre los principales estudios y evaluaciones realizadas se tienen (Aguinaga, 2006):

- En 1975, Minero Perú: exploración preliminar de manifestaciones geotermales de Calacoa y Salinas (Moquegua).

- En 1976, Geothermal Energy Research del Japón: exploraciones preliminares en la cuenca del Vilcanota (Cusco).

- En 1977, INIE: primer censo de manifestaciones geotermales.

- En 1978, INGEMMET: inventario y una agrupación geográfica de afloramientos geotermales (se definieron las Regiones Geotermales).

- Entre 1979 y 1980, INGEMMET y Aquater de Italia: estudios de reconocimiento geotérmico de Región V (identificando áreas de interés: Tutupaca, Calacoa, Challapalca, Salinas, Chachani y Chivay).

- En 1980, Geothermal Energy System Ltd.: estudios de reconocimiento geotérmico en las zonas de Calacoa, Tutupaca y Salinas (Moquegua).

- Entre 1983 y 1985, INGEMMET y British Geological Survey: inventario parcial de manifestaciones geotermales en Región VI (Cusco y Puno).

- Entre 1983 y 1986, ELECTROPERÚ y Cesen de Italia: estudios de reconocimiento geotérmico en Regiones I a IV; y estudio de prefactibilidad en La Gramma (Cajamarca).

- En 1986, ELECTROPERÚ con asistencia técnica de IAEA y ONU: investigaciones geoquímicas en la Región $\mathrm{V}$ (Tacna y Moquegua).

- En 1997, CENERGÍA, con apoyo del IIE de México: evaluación de información y estudios disponibles (realizados por INGEMMET, ELECTROPERU, Proyecto Especial Tacna, IPEN y la Cooperación Internacional).

En base a los estudios anteriores, realizados en unos $100 \mathrm{mil} \mathrm{km}^{2}$, el SNL (Battocletti, 1999) estimaría que el potencial geotérmico del Perú 
se encontraría entre 1000 a 2990 MW. En el 2008, quedó pendiente de formalización la oferta del Gobierno del Japón de apoyar al país con el plan maestro de Geotermia. Este programa de cooperación técnica debería reactivarse.

\section{Hidroelectricidad}

El agua es la principal energía renovable del Perú, habiéndose desarrollado un conjunto de centrales hidroeléctricas que han significado importante ahorro de recursos y menor contaminación para el país. Además, dentro de los varios tipos de centrales, el tipo que se ha usado más en el país, es aceptada, dependiendo del caso específico, dentro del mecanismo de desarrollo limpio.

En la década del 70, en el marco del Programa de Cooperación Energética Peruano-Alemana, se realizó una Evaluación del potencial hidroeléctrico nacional (GTZ \& LIS, 1979), evaluándose alrededor de 800 proyectos hidroeléctricos con una potencia mínima de $30 \mathrm{MW}$, y seleccionándose finalmente 328 proyectos hidroeléctricos que cumplían los criterios de viabilidad definidos. Este conjunto de proyectos sumaba una potencia instalada promedio de $58937 \mathrm{MW}$ y una energía anual acumulada de 395118 GW.h, la cual es considerada hasta la actualidad como el potencial hidroeléctrico nacional técnicamente aprovechable, si bien no se dispone de información actualizada sobre el potencial hidroeléctrico.

En el Perú, para el desarrollo de la actividad de generación hidroeléctrica, el Ministerio de Energía y Minas (MEM) otorga el derecho eléctrico de concesión. Conforme al Decreto Ley $\mathrm{N}^{\circ}$ 25844, Ley de Concesiones Eléctricas (LCE), el MEM otorga concesiones temporales para el desarrollo de estudios hasta nivel de factibilidad, y concesiones definitivas para la ejecución de proyectos hidroeléctricos. La concesión temporal no tiene carácter de exclusividad, mientras que la concesión definitiva si es exclusiva.

\section{Barreras y avances}

A pesar del alto potencial hidroeléctrico que tiene el Perú, en los últimos 12 años el crecimiento de la oferta de Potencia Efectiva de generación en centrales hidroeléctricas ha sido de tan solo $322 \mathrm{MW}$, mientras que en centrales termoeléctricas a gas natural el crecimiento ha sido de 1249 MW. Casi la totalidad de la capacidad instalada en centrales hidroeléctricas en el SEIN ha sido construida por el Estado, y luego varias de éstas han sido privatizadas a partir de la década del 90.

Las principales barreras para el desarrollo de proyectos hidroeléctricos, en comparación con otras tecnologías, como las centrales térmicas a gas natural, son las siguientes:

a) Montos de Inversión

Las centrales hidroeléctricas se caracterizan por tener bajos costos de producción (etapa de operación), pero muy altos los costos de inversión (etapa de construcción), en comparación con otros tipos de tecnologías. Por cada 
MW de potencia instalada, se requiere una inversión entre 1,2 a 1,8 millones US\$; y para una termoeléctrica a gas natural, en ciclo simple, por ejemplo, se requiere un monto promedio de inversión de 0,5 millones US\$ por cada MW instalados, que incluso se facilita bajo la modalidad de leasing.

b) Periodos de Construcción

Ciertamente, las centrales hidroeléctricas de gran tamaño tienen un período de construcción que en promedio es de 4 a 5 años, mientras una termoeléctrica a gas natural tiene un periodo de construcción de un año a año y medio, aproximadamente. Estos mayores plazos implican mayores gastos financieros durante la etapa de construcción de una central hidroeléctrica, que tendría ingresos recién al sexto año, mientras que la termoeléctrica los tendría antes del segundo año.

c) Financiamiento

La principal dificultad para lograr el financiamiento, es el no contar con un contrato a largo plazo por la venta de la energía a un precio fijo, de tal manera que tenga garantizado un flujo de capital que asegure el retorno de la inversión. La conjunción de este aspecto con los dos anteriores, en definitiva, ha determinado que en el Perú la inversión privada se oriente a la construcción de centrales térmicas a gas natural en lugar de centrales hidroeléctricas. Pues requiere de menores montos de inversión, y por su menor periodo de construcción genera ingresos cuatro años antes que en el caso de una hidroeléctrica. d) Tarifas en Barra y Precio del Gas Natural

Complementariamente, conforme a las disposiciones vigentes en el Marco Regulatorio hasta diciembre de 2004, desde el año 2001 las Tarifas en Barra, a las cuales los generadores podian contratar la venta de su energía a los distribuidores para el mercado regulado, han sido fijadas considerando el precio promocional del gas natural de Camisea para los generadores eléctricos, determinándose así tarifas ciertamente bajas, no eran suficientes para incentivar la inversión en centrales hidroeléctricas. Vale recordar que la primera central termoeléctrica a gas natural de Camisea empezó recién a operar en agosto de 2004. Es así que el precio del gas natural, indirectamente también ha sido una variable que ha venido limitando el desarrollo de centrales hidroeléctricas.

Resulta necesario precisar que el precio del gas natural proveniente de Camisea, es un caso particular, se trata de un yacimiento ya descubierto por la Shell, por lo que los costos de inversión en exploración para el productor fueron cero, ello complementado a que se trata de un yacimiento con alta composición de condensados, por lo que el principal negocio son los líquidos extraídos. Es así que cerca del 80\% de los ingresos del productor corresponden a la venta de los liquidos.

Es por ello que todo aumento del costo del gas natural a los generadores termoeléctricos, puede aplicarse vía no remuneración de la potencia por 
uso ineficiente del gas en ciclo simple o un impuesto al consumo de dicho combustible. Sus fondos deberían ser destinados a financiar estudios, hasta nivel de pre factibilidad, de proyectos hidroeléctricos y otras renovables, a fin de que el Estado mantenga una cartera de proyectos licitables y así se reduzca el riesgo del inversionista, reforzando su rol promotor de la inversión.

Identificadas las barreras para el desarrollo hidroeléctrico, desde el año 2006 ya se han dictado normas legales orientadas a promover la inversión en centrales hidroeléctricas, como un claro avance para contrarrestar las barreras que limitan el mejor aprovechamiento del alto potencial hidroeléctrico que dispone el país, sobre todo aquellos proyectos cuyo impacto ambiental es mitigable.

e) Marco Normativo promotor

En julio de 2006 se dio la Ley $N^{\circ}$ 28832, Ley para Asegurar el Desarrollo Eficiente de la Generación Eléctrica, complementando el Marco Legal del Sector Eléctrico. Conforme al Marco Legal del Sector Eléctrico Peruano, todo Generador puede comercializar su producción de energía bajo cuatro (04) modalidades:

1. Contratos con Distribuidores, a través de las Licitaciones de Suministro de Electricidad para el Mercado Regulado y/o Libre del Distribuidor. El precio tiene carácter de firme y es el correspondiente a la oferta de cada Generador.

2. Contratos con Distribuidores para su Mercado Regulado. El precio corresponde a la Tarifa en Barra que fija OSINERGMIN.

3. Contrato con Usuarios Libres a precio negociado.

4. Transferencias en el Mercado de Corto Plazo administradas por el COES.

El precio corresponde al spot o Costo Marginal de corto plazo, mecanismo con el cual se repaga la energía tomada de la red y no generada para cubrir por el generador los contratos de sus clientes contratados.

De estas modalidades, la tendencia es que la b) se reduzca sustancialmente, y que la atención de la totalidad de la demanda del Mercado Regulado sea abastecida a través de las Licitaciones de Suministro de Electricidad. Es justamente la modalidad Licitaciones de Suministro de Electricidad, la más adecuada para lograr viabilizar la inversión en una central de generación, debido a los contratos de suministro de largo plazo (de hasta 20 años) que se pueden obtener y que facilitarán la obtención del financiamiento correspondiente.

La Ley $\mathrm{N}^{\circ} 28832$ crea el mecanismo de Licitaciones de Suministro de Electricidad convocadas por los Distribuidores, con el objetivo de reducir la intervención administrativa en la determinación de precios de generación mediante soluciones de mercado, a fin de promover una efectiva competencia y nuevas inversiones en generación.

En cada licitación las Bases son propuestas por los Distribuidores y aprobadas por OSINERGMIN, quién también fija un Precio Tope 
escondido de Adjudicación para dicha licitación. El precio de venta de energía a los Distribuidores es el ofertado por cada postor, el precio de potencia corresponde al Precio Básico de Potencia (que fija OSINERGMIN) vigente a la fecha de la convocatoria de la licitación. Ambos precios tienen carácter de firmes durante el periodo contractual (hasta por 20 años) y se actualizan durante la vigencia del contrato aplicando las correspondientes fórmulas de actualización establecidas en las propias Bases de la licitación.

La mencionada Ley $\mathrm{N}^{\circ} 28832$, establece que en las licitaciones se debe aplicar un factor de descuento a las ofertas económicas respaldadas con proyectos hidroeléctricos para efectos de su evaluación, toda vez que esos proyectos implican mayores costos de inversión que los proyectos térmicos. La aplicación de dichos factores de descuento es únicamente para efectos de la evaluación de la oferta económica, pues todo postor que se convierta en adjudicatario siempre recibirá por la venta de su energía el precio que hayan ofertado en la licitación.

El Cuadro $N^{\circ} 01$ resume los tipos de licitaciones contempladas en la referida Ley $\mathrm{N}^{\circ} 28832$ :

\section{Cuadro $\mathbf{N}^{\circ} 1$ Tipos de Licitaciones de Suministro de Electricidad}

(Ley 28832)

\begin{tabular}{|c|c|c|c|}
\hline Marco Legal & Anticipación & Periodo & Demanda* \\
\hline Marco General & $\begin{array}{c}3 \text { años** } \\
3 \text { años } \\
\text { menor a 3 años }\end{array}$ & $\begin{array}{c}\text { Largo (hasta 20 años) } \\
\text { Mediano (hasta 5 años) } \\
\text { Mediano / Largo }\end{array}$ & $\begin{array}{c}\text { hasta 100\% } \\
\text { hasta 25\% } \\
\text { Hasta 10\% }\end{array}$ \\
\hline $\begin{array}{c}\text { 4ta DCT } \\
\text { (hasta jul. 2009) }\end{array}$ & menor a 3 años & Mediano (hasta 5 años) & hasta 100\% \\
\hline
\end{tabular}

(*) del Mercado Regulado

$\left.{ }^{* *}\right)$ Los Distribuidores tienen el incentivo para que liciten con mayor anticipación, hasta de 6 años, permitiéndose trasladar a la demanda el precio resultante de las licitaciones con un incremento de hasta $3 \%$

Fuente: MINEM.

f) Incentivos Tributarios

Adicionalmente, se han dictado leyes que otorgan beneficios tributarios a la inversión en renovables, como son:

i. Ley $\mathrm{N}^{\circ} 28876$, dispone que la generación de energía eléctrica con recursos hidráulicos y otros renovables, pueden acogerse al Régimen de Recuperación Anticipada del Impuesto General a las Ventas (IGV).

ii. Decreto Ley $\mathrm{N}^{\circ}$ 1058, que dispone el beneficio de la depreciación acelerada de 
activos, de hasta $20 \%$ cada año, para efectos del pago del Impuesto a la Renta, para las inversiones en hidroeléctricas y demás energías renovables.

\section{g) Medidas Pendientes}

Es importante el avance para incentivar la inversión en centrales hidroeléctricas y otras energias renovables. Sin embargo, aún hay medidas pendientes de adoptar para promover el desarrollo sostenible y la diversificación de la matriz energética, a fin de coadyuvar hacia el abastecimiento seguro, oportuno y eficiente de la demanda de energía eléctrica. Entre estas se puede mencionar:

i. Rol planificador del Estado, que prevea en el largo plazo el nivel de participación de cada tecnología en la oferta de generación, entre ellas las renovables, como la hidroeléctrica.

ii. A fin de garantizar la implementación de dicho planeamiento, debe ser el Estado quien decida la oportunidad en que se convoquen las Licitaciones de Suministro de Electricidad de largo plazo. E1 estado debe hacer licitaciones por mayores demandas de energía, promoviendo una mayor competencia, y orientando las inversiones en nueva oferta de generación hacia un crecimiento descentralizado y distinguiendo por tecnologías y cuál va a ser priorizada. Para ello, los distribuidores comunicarian sus requerimientos futuros de energía.

iii. El Perú presenta naturalmente una estructura de consumo y generación seccionada en no menos de tres grandes macroregiones, norte, centro y sur; hacia ellas debiera dirigirse la solicitud o llamado a licitación para proveer energía renovable, así el efecto de localización jugaría del lado correcto para mantener racional los costos de la red de transmisión eléctrica nacional.

iv. Como medida complementaria a los puntos mencionados, se debe perfeccionar la Cuarta Disposición Complementaria Fina de la Ley $N^{\circ}$ 28832. Esta Ley dispone que el Ministerio de Energía y Minas debe poner a disposición de los futuros inversionistas proyectos con estudios hasta nivel de prefactibilidad, estableciendo que los estudios sean hasta nivel de factibilidad y eventualmente con los correspondientes estudios de impacto ambiental; ello con la finalidad de reducir los riesgos al inversionista $\mathrm{y}$ para que así el Estado tenga un rol más promotor o facilitador.

v. Bajo el esquema de contratos de venta de energía hasta por 20 años a precios firmes actualizables, como resultado 
de Licitaciones especiales de Suministro de Electricidad, los proyectos serian viables y conseguirian financiamiento fácilmente, por lo que el tema del bajo precio del gas natural del lote 88 para los generadores ya no sería una barrera. Sin embargo, a pesar de no ser tema directo del presente documento, la aplicación de un impuesto al consumo ineficiente de dicho combustible para su uso eléctrico se considera una medida necesaria y pendiente para promover de manera efectiva el uso eficiente en la generación eléctrica a través de la tecnología del ciclo combinado. Otra medida ya está en el Decreto Legislativo 1041, que inexplicablemente fue suspendido por un Decreto de Urgencia. En el 1041, la remuneración por potencia, que es un componente de la tarifa, solo procede si hay un uso eficiente del gas natural.

Un motivo de reflexión es aprender de nuestros errores como país y como el resultado poco satisfactorio en la primera licitación de largo plazo de hidroeléctricas que convocó PROINVERSION, por la insistencia en mantener un precio que no corresponde al mercado y la falta de proyectos con estudios suficientes. Error que se corrigió con una nueva convocatoria, que consiguió un resultado positivo mejor. Además, no se permitió la participación de asociaciones público privadas, inexplicablemente, se redujo el concurso de 1,500 MW, requeridos por el ex Ministro Valdivia a $500 \mathrm{MW}$, por decisión del Ministro Sánchez. La consecuencia fue que se comprometió el crecimiento eléctrico a un mayor consumo de hidrocarburos. Esto perjudica en el largo plazo la competitividad y sostenibilidad de la economía peruana.

Una buena señal ha sido la primera subasta de energías alternativas, que no obstante su retraso, ha marcado un hito. Se ha llevado a cabo la primera Subasta de suministro de electricidad con Recursos Energéticos Renovables (RER), en el marco del Decreto Legislativo $\mathrm{N}^{\circ} 1002$, "Ley de promoción de la inversión para la generación de electricidad con el uso de energias renovables", y su Reglamento aprobado por Decreto Supremo N ${ }^{\circ}$ 050-2008-EM. Conforme al referido marco legal, el Ministerio de Energía y Minas (MEM) elaboró las Bases de la Subasta, encargándose OSINERGMIM de la conducción del proceso. Dicho proceso se inició el 21 de agosto de 2009 con la publicación del Aviso Previo por parte del MEM y la apertura del Registro de Participantes en el Portal Web de OSINERGMIN, quién efectúo la correspondiente convocatoria el 15 de octubre de 2009.

La presentación de sobres técnicos y económicos se efectuó el 18 de enero de 2010, publicándose la relación de Postores calificados el 29 de enero de 2010. El 12 de febrero de 2010 se llevó a cabo el Acto Público 
de apertura de sobres económicos y otorgamiento de la Buena Pro de la primera Subasta RER. A esta etapa calificaron veinte (20) Postores con treinta y un (31) proyectos; 17 proyectos hidroeléctricos, 06 eólicos, 02 de biomasa, y 06 solares.

Finalmente, los proyectos adjudicados han sido 26. Los Precios Máximos de Adjudicación fijados por OSINERGMIN para las tecnologías hidroeléctrica, eólica, biomasa, y solar fueron de 74 US\$/MWh, 110 US\$/ MWh, 120 US\$/MWh, y 269 US\$/ MWh, respectivamente. Estos precios fueron mantenidos en reserva por el Notario Público, y dados a conocer al inicio del Acto Público de apertura de sobres económicos y otorgamiento de la Buena Pro. La media de los precios adjudicados (ofertados por los Postores adjudicatarios) fue como sigue:

\begin{tabular}{|c|c|c|c|}
\hline Tecnologias & \multicolumn{3}{|c|}{ Precios } \\
\hline Hidro & $\begin{array}{l}\text { 60,2 US\$/ } \\
\text { MWh }\end{array}$ & $\begin{array}{l}\text { con un minimo de 55,0 } \\
\text { US } \$ \text { / MWh }\end{array}$ & $\begin{array}{l}\text { y un máximo de } 70,0 \\
\text { US } \$ \text { /MWh }\end{array}$ \\
\hline Eólica & $\begin{array}{l}80,36 \text { US\$/ } \\
\text { MWh }\end{array}$ & $\begin{array}{l}\text { con un minimo de } 65,5 \\
\text { US } \$ \text { / MWh }\end{array}$ & $\begin{array}{l}\text { y un máximo de } 87,0 \\
\text { US } \$ / M W h\end{array}$ \\
\hline Biomasa & $\begin{array}{l}\text { 80,36 US\$/ } \\
\text { MWh }\end{array}$ & $\begin{array}{l}\text { con un mínimo de } 52 \\
\text { US } \$ / M W h\end{array}$ & $\begin{array}{l}\text { y un máximo de } 110 \\
\text { US\$ / MWh }\end{array}$ \\
\hline Solar & $\begin{array}{l}\text { 221,09 US\$/ } \\
\text { MWh }\end{array}$ & $\begin{array}{l}\text { con un minimo de } 215 \\
\text { US\$ / MWh }\end{array}$ & $\begin{array}{l}\text { y un máximo de } 225 \\
\text { US } \$ \text { /MWh }\end{array}$ \\
\hline
\end{tabular}

Las potencias adjudicadas corresponden a 162,3 MW hidroeléctricos, $142 \mathrm{MW}$ eólicos, 27,4 MW biomasa, y 80 MW solares, haciendo un total de 411,7 MW adjudicados. Las correspondientes energías adjudicadas son de 999,3 GWh/año a las hidroeléctricas, y $887,3 \mathrm{GWh} /$ año a las otras energías renovables no convencionales (eólica/ biomasa/solar). En sintesis, se ha adjudicado una potencia total de 411,7 MW, que corresponde a una energía total de 1 886,6 GWh/año, a un precio medio de adjudicación de 81,2 US\$/MWh... Conforme al cronograma establecido en las Bases, el 31 de marzo de 2010, se estableció la firma con los Postores adjudicatarios los correspondientes los contratos de suministro de electricidad con RER por un plazo de 20 años.

Debemos trabajar por una matriz energética que ayude, en el mediano y largo plazo, al crecimiento sostenible del país y a mitigar los efectos del cambio climático. Por ello, debemos usar más y mejor el agua, los vientos, la geotermia y orientar el uso del gas disponible -que es un recurso no renovable y con reservas limitadas- al transporte, viviendas, industria, y usar la tecnología del ciclo combinado en generación eléctrica, es decir, un uso eficiente del gas, con valor agregado. No olvidemos 
que el gas del lote 88 con precio promotor y regulado, solo alcanza, al actual nivel de crecimiento, para atender la demanda nacional hasta el 2019. Después deberá utilizarse gradualmente para completar parte de la atención de la demanda local, el gas de otros lotes, a precio internacional. Por ello fue un error del ex Ministro Sánchez que parte del gas del lote 57 se orientara al mercado interno, cuando el compromiso original de Repsol, en el 2008, manifestado en carta de su principal directivo, era utilizar este nuevo descubrimiento para liberar el gas del lote 88, del compromiso de exportación. Esto, finalmente fue definido positivamente por el actual gobierno, que negoció la restitución del lote 88 para el mercado interno.

Las Distribuidoras del Estado deberian comprometer hasta donde sea posible, su demanda futura de electricidad, que en las licitaciones de largo plazo es hasta 20 años, en las subastas especiales para hidroeléctricas $\mathrm{y}$, en segundo lugar, en la licitación de otras energías renovables. Si participan en las llamadas licitaciones ordinarias que no son especiales para hidroeléctricas o las de otras renovables, se favorece que haya más generación térmica, es decir, más demanda y consumo de hidrocarburos. Es muy dificil que puedan competir las hidroeléctricas, por los significativos mayores montos de inversión, mayor tiempo de construcción y recuperación de la inversión, no obstante el factor de descuento de 15\%. Comprometer la demanda eléctrica en el largo plazo a un horizonte térmico no es lo mejor para el país, porque esa dependencia afecta el futuro y competitividad de la minería, la industria y la economía nacional. 


\section{REFERENCIAS Y BIBLIOGRAFÍA RECOMENDADA}

AGUINAGA J. (2006). Situación de la geotermia en el Perú. Lima: MEM, Dirección General de Electricidad.

ATLAS DE ENERGÍA SOLAR DEL PERÚ (SENAMHI, 2003).

BANCO MUNDIAL. Análisis Ambiental del Perú: Retos para un Desarrollo Sostenible. Resumen Ejecutivo. Mayo 2007.

BATTOCLETTI L. (1999). Geothermal resources in Latin America \& the Caribbean. Livermore: SNL, Sandia's California Laboratory.

CALVO, Eduardo (2008). Inventario Integrado de Emisiones de Gases de Efecto Invernadero del Perú en el año 2000. Informe preparado para el Ministerio del Ambiente en el marco del proyecto "Segunda Comunicación Nacional del Perú a la Convención del Marco de las Naciones Unidas sobre Cambio Climático". Marzo 2009

CENTRO DE CONSERVACIÓN DE ENERGÍA Y DEL AMBIENTE (CENERGIA) (2004). Diagnóstico de la situación actual del uso de la energía solar y eólica en el Perú. Lima: MEM.

CENTRO DE ENERGÍAS RENOVABLES Y USO RACIONAL DE LA ENERGÍA DE LA UNIVERSIDAD NACIONAL DE INGENIERÍA (CER UNI) (2005a). Estudio sobre la situación actual de las energias renovables del país y su perspectiva de desarrollo en el mercado energético nacional. Informe Final. Lima: FONAM.

CENTRO DE ENERGÍAS RENOVABLES Y USO RACIONAL DE LA ENERGÍA DE LA UNIVERSIDAD NACIONAL DE INGENIERÍA (CER UNI) (2005b). Diagnóstico de las instalaciones fotovoltaicas y elaboración de propuesta normativa. Lima: OSINERG.

COMISIÓN EUROPEA, Informe de Abril de 2009, "The impact of renewable energy policy on economic growth and employment".

COVIELLO M. (2006). CEPAL y la geotermia en el Perú. Santiago de Chile: CEPAL, División de Recursos Naturales e Infraestructura.

GAMIO Pedro. Cambio de Matriz Energética y Politicas Públicas para las Energias Renovables y los Biocombustibles. Presentación del Viceministro de Energía en el II Congreso Nacional de Energías Renovables y Biocombustibles. 25-28 de octubre de 2008.

GAMIO Pedro. Apuntes sobre politica energética. Presentación realizada en la Cumbre América Latina y Europa, ALCUE. 13 -17 Mayo 2008.

GREEN ENERGY. (2005). Estudio para la promoción de la generación eléctrica con fuentes de energía renovable. Lima: MINEM, Dirección General de Electricidad.

HORN, M. (2007). Potencial de energía solar térmica y fotovoltaica en el Perú. Presentación en el I Congreso sobre Biocombustibles y Energias Renovables. Lima: 17-19 de mayo del 2007, Universidad Nacional Agraria La Molina. 
MAYORGA E. (2007). Potencial del viento y la aerogeneración en el Perú. Presentación en el I Congreso sobre Biocombustibles y Energias Renovables. Lima: 17-19 de mayo del 2007, Universidad Nacional Agraria La Molina.

MEM, 1979. Evaluación del Potencial Hidroeléctrico Nacional. Ministerio de Energía y Minas - Dirección General de Electricidad, Documento elaborado con el apoyo de la Sociedad Alemana de Cooperación Técnica (GTZ) y el consorcio Lahmeyer. Salzgitter.

MEM, 2008. Balance Nacional de Energía 2007. Ministerio de Energía y Minas, Oficina de Planeamiento, Inversiones y Cooperación Internacional.

MEM. Atlas Eólico del Perú. Ministerio de Energía y Minas. Noviembre 2008.

MEM, 2009. Propuesta de Estrategia para Mitigar las Emisiones de Gases de Efecto Invernadero en los sectores Energia, Industria y Transporte 2008-2050. Informe Final. Ministerio de Energía y Minas 2009.

MINAM, 2009. Sembremos para el Futuro. Peruanos trabajando juntos por el Ambiente. Ministerio del Ambiente. 1er Aniversario. 14 de mayo 2009.

OIT, Set. 2008. "Información sobre los empleos verdes y la Iniciativa Empleos Verdes": http://www.ilo.org/integration/greenjobs/index.htm

OLAZÁBAL, J. Potencial hidroeléctrico nacional. Presentación en el I Congreso sobre Biocombustibles y Energías Renovables. Lima: 17-19 de mayo del 2007, Universidad Nacional Agraria La Molina.

OSINERGMIN, 2009. "Resultados del Control de Azufre y Plomo en Plantas y Refinerías - Período 2008-II": (http://www.osinerg.gob.pe/newweb/uploads/ GFH/Calidad/RESULTADOSDECALIDADENPLANTASYREFINERIAS(2008-II). pdf) junio del 2009.

PRODUCE, 2007. Censo Nacional de Establecimientos Manufactureros 2007, Ministerio de la Producción, Vice ministerio del MYPE e Industria. Según información presentada en su página web a junio de 2009.

RÁEZ LUNA, Ernesto, Exposición en el Simposio de la UPCH "Desarrollo Hidroeléctrico Sostenible en la Amazonia y el caso de la cuenca del río Madeira (Bolivia, Brasil, Perú)". Lima, 25 y 26 de agosto de 2009

SECRETARIA GENERAL DE LA COMUNIDAD ANDINA (2008). El Cambio Climático no tiene fronteras: Impacto del Cambio Climático en la Comunidad Andina.

VELÁSQUEZ J. (2007). Mapa Eólico Preliminar del Perú. Lima: ADINELSA. 